Martínez-Sánchez, A.M., Esteban-Moreno, R.M. \& Pabón-Carrasco, M. (2022). Análisis de las actitudes hacia el hecho multicultural en una muestra de estudiantes españoles de Magisterio. Revista Electrónica Interuniversitaria de Formación del Profesorado, 25(1), $19-34$.

DOI: https://doi.org/10.6018/reifop.496431

\title{
Análisis de las actitudes hacia el hecho multicultural en una muestra de estudiantes españoles de Magisterio
}

Alina de las Mercedes Martínez Sánchez ${ }^{(1)}$, Rosa María Esteban Moreno ${ }^{(2)}$, Manuel Pabón Carrasco $^{(3)}$

${ }^{(1,2)}$ Universidad Autónoma de Madrid, ${ }^{(3)}$ Universidad de Sevilla

\section{Resumen}

La multiculturalidad es un hecho que tiene su impacto en el sistema educativo y demanda atender a la formación del profesorado para educar en la diversidad. Se desarrolló una investigación con enfoque cuantitativo apoyado en un único método de recogida de datos con carácter de escala ordinal tipo Likert, basado en un diseño no experimental, exploratorio, longitudinal y descriptivo. Para obtener la información se aplicó un cuestionario (Escala de Actitudes hacia el Hecho Multicultural) con un Alpha de Cronbach de 0,8 a una muestra de 1.170 estudiantes de magisterio. Aunque aparecen actitudes positivas hacia el hecho multicultural, se constata la necesidad de trabajar, desde las Facultades de Educación, la educación multicultural, que facilite a los futuros maestros la incorporación, en su actividad diaria, de una respuesta acorde con el hecho multicultural de nuestro país.

\section{Palabras clave}

Actitudes; multiculturalidad; profesores; educación; escala Likert.

\section{Contacto:}

Alina de las Mercedes Martínez Sánchez, alina.martinez@uam.es, Facultad de Educación, Universidad Autónoma de Madrid, Campus Cantoblanco, Avenida Tomás y Valiente s/n - 28049 Madrid (España). 


\title{
Analysis of attitudes towards multiculturalism in a sample of Spanish teacher training students
}

\begin{abstract}
Multiculturalism is a fact that has its impact on the educational system and demands attention to the training of teachers to educate in diversity. Research with a quantitative approach was developed based on a single data collection method with an ordinal Likert scale character, based on a non-experimental, exploratory, longitudinal, and descriptive design. To obtain the information of applied a questionnaire (Scale of Attitudes towards the Multicultural Fact) with an Alpha of Cronbach of 0.8 to a sample of 1,170 students of teaching. Although positive attitudes towards the multicultural fact appear, it is verified the necessity to work, from the Faculties of Education, the multicultural education, that facilitates to the future teachers the incorporation, in its daily activity, of an agreed answer with the multicultural fact of our country.
\end{abstract}

\section{Key words}

Attitudes; multiculturalism; teachers; education; Likert scale.

\section{Introducción}

La diversidad cultural es un hecho que marca a las sociedades actuales a nivel global y España se presenta como una muestra en sí misma. En enero de 2021, el Instituto Nacional de Estadística (INE, 2021) recoge que la población española supera los 47 millones de habitantes. Según datos publicados por la Organización de Naciones Unidas (ONU, 2019), el 12,96\% de la población de España es inmigrante, siendo el país $46^{\circ}$ del mundo por porcentaje de inmigración (datosmacro.com, 2019). En este concierto, Bayona (2019) señala que ya en 2018 los hijos de padres extranjeros ascienden a 7,82 millones de personas. Estos datos ponen de relieve la magnitud del fenómeno migratorio en España permitiendo definirla como un país multicultural en el cual emerge el imperativo de la integración del "otro".

La literatura es prolífera en la presentación de definiciones del término multiculturalidad. Olivé (2004) refiere la multiculturalidad como un término para describir sociedades donde conviven grupos procedentes de diversas culturas, identificándolo al mismo tiempo con la pluriculturalidad. Para Requejo (2010) se trata de un concepto que traslada al carácter culturalmente heterogéneo de las personas que conviven en una sociedad. Desde las perspectivas de la sociología y la antropología cultural, la multiculturalidad se refiere a la existencia de diferentes culturas en un mismo espacio geográfico y social, y, aunque cohabitan, esto no lleva implícito ningún intercambio ni profundas influencias entre ellas (Bernabé, 2011).

En virtud de que la multiculturalidad no presupone contacto social entre culturas, en este trabajo se reconoce la diferencia de este concepto con el término interculturalidad, en el cual se pone la mirada en la interacción, el encuentro y la relación entre culturas sobre bases de respeto (Arroyo, 2013).

En contraste, el multiculturalismo, término surgido en la segunda mitad del siglo XX en países anglosajones como Canadá y Estados Unidos, es utilizado para referir un conjunto de cuestiones que engloban la existencia de múltiples culturas, la ideología de respeto y 
convivencia en el contexto de políticas implementadas por los gobiernos, sobre todo en relación con los inmigrantes (Barabas, 2014).

Se trata así, de un proyecto normativo para la regulación de la convivencia entre culturas (Hernández, 2007). Supone que hay una suma en el tiempo y el espacio de grupos étnicos de diferentes orígenes; acepta y reconoce la diversidad de culturas, pero no establece vínculos reales entre la cultura de referencia y las culturas de los ciudadanos de otros orígenes. Estrach (2001) considera que las reflexiones sobre el multiculturalismo deben ser críticas en tanto que, si bien reconoce las diferencias culturales y destaca la importancia de la afirmación de las creencias particulares y diferenciadas, termina por atender de manera exclusiva a las contingencias y al folklore, olvidando las necesidades reales que engendra la convivencia ciudadana de la diversidad cultural en la política.

La multiculturalidad es un hecho que tiene su impacto en el sistema educativo y atender a la formación del profesorado para educar en la diversidad, demanda la necesidad de conocer los distintos elementos que subyacen en las actitudes asumidas por los seres humanos frente a las diferencias, en tanto que, no siempre son positivas las expectativas que el profesorado elabora sobre el alumnado (Intxausti, 2014).

En su acepción más clásica, Allport (1935) define la actitud como un estado de disposición mental y nerviosa, organizado mediante la experiencia, que ejerce un influjo directivo dinámico en la respuesta del individuo a toda clase de objetos y situaciones. Según Montes (2008), la actitud es el resultado del proceso de socialización y tiene una gran influencia en la conducta poseyendo fundamentalmente un componente evaluativo. En ambas definiciones se pone de manifiesto que la actitud es una disposición previa y preparatoria a respuestas conductuales ante estímulos sociales. En resumen, se trata de un concepto complejo que involucra procesos motivacionales relativos a los sentimientos o emociones; procesos cognitivos que remiten a las formas de percepción de un objeto y que con frecuencia es un estereotipo; y procesos conductuales que engloban la tendencia o disposición a la actuación frente a un objeto o situación referida al mundo del individuo (Casas, 1999; Escalante, Repetto y Martinello, 2012 y Padua, 1979). El conocimiento y evaluación de todas estas dimensiones serán un elemento clave en el proceso de modificación de las actitudes hacia el hecho multicultural.

El sistema educativo español encara el desafío de tener en la actualidad altas tasas de estudiantes de nacionalidad extranjera como resultado de la inmigración, lo cual convierte las escuelas en verdaderos escenarios multiculturales.

Este panorama educativo cohabita con la realidad de una población inmigrante con una tasa de paro del $25,1 \%$ frente a una del $14,1 \%$ para los nacionales sólo españoles en 2018 . El 27,9 por 100 del alumnado extranjero abandona el sistema escolar sin alcanzar el título de educación secundaria obligatoria, frente al 6,3\% del de nacionalidad española (Consejo Económico y Social, 2019); el bullying que sufren los hijos de emigrantes ha aumentado en casi tres puntos entre 2015 y 2017, y roza ya el 10\% del total de los casos de acoso escolar (Casares, 2019).

Numerosos reportes indican que el racismo es una cuestión no superada en España. MoldesMaya (2018) refiere la existencia de actitudes negativas hacia la inmigración en la población española, relacionada con elementos causales sustentados en amenazas-económico materiales como factor detonante, resultados coincidentes con los análisis de opinión sobre inmigrantes en la población española realizados por Rinken (2015).

Los estudios de Segura y Gallardo (2017) muestran la existencia de prejuicios sutiles y manifiestos en jóvenes españoles respecto a los inmigrantes. Assiego (2016) señala que el $48 \%$ de la población española piensa que los inmigrantes contribuyen poco o el $64 \%$, nada, a 
la creación de empleo. La cifra de "delitos de odio" registrada por las Fuerzas y Cuerpos de Seguridad en España, a lo largo de 2018, asciende a un total de 1.598 incidentes, lo que supone un aumento del 12,6\% en relación con 2017. Entre los tres ámbitos que mayor número de incidentes registran se encuentran los de "ideología", "racismo/xenofobia" y "orientación sexual e identidad de género" que representan del total del conjunto de delitos de odio el 81,8\% (Ministerio del Interior, 2020).

En 2015, la Organización para la Cooperación y el Desarrollo Económicos (OCDE) suspende a España en materia de igualdad en el plano educativo (CEAR, 2016). A pesar de los fundamentos presentados por el Informe PISA de 2015 refiriendo al factor económico como causal de los 26 puntos menos obtenidos por los estudiantes inmigrantes, respecto a los nacidos en España, el 35,7\% de los españoles sigue pensando que la calidad de la educación empeora en los colegios donde hay muchos hijos de inmigrantes, según encuesta del Centro de Investigaciones Sociológicas (CIS) (Carmona, 2017).

La implicación y actitud del profesorado en el tratamiento pedagógico a la realidad culturalmente diversa ha sido objeto de estudio por diversos autores al evidenciar la necesidad de que los docentes manifiesten actitudes positivas que favorezcan la educación intercultural. Bagan, Giménez, Monserrat, y Ruiz (2005) describen la existencia de actitudes negativas hacia el hecho multicultural en los profesores, a medida que se elevan los niveles o etapas donde se imparte la docencia e Iñiguez-Berrozpe, Valero, Flecha y Redondo-Sama (2021) concluyen en su estudio que los enfoques de cómo plasmar la educación intercultural en las aulas se queda en la superficie en el profesorado en formación.

Estudios realizados por Rodríguez y Fernández (2018) dan cuenta del impacto del desconocimiento de docentes y discentes sobre otras culturas y corroboran el impacto de las actitudes del profesorado en los peores rendimientos del alumnado de otras culturas. Se constata la presencia de actitudes de insensibilidad hacia los modelos de educación intercultural, al evaluar las actitudes en función de la formación de los docentes (González, Berríos y Buxarrais, 2013).

La comprensión de la diversidad en su dimensión ética implica una reflexión sobre la disposición a defender los derechos de los "otros" como legítimos (Bazán, 2011). En esta dirección, la formación del profesorado se convierte en elemento clave para la gestión de los conflictos derivados de actitudes negativas ante el aprendizaje o de prejuicios culturales que impliquen discriminación (Figueredo-Canosa, 2017).

La Agenda 2030 aspira a garantizar una educación inclusiva y equitativa de calidad y promover oportunidades de aprendizaje permanente para todos, por lo que es necesario replantearse el proceso de formación del profesorado (Herrera y Blanco, 2018), aspiración manifiesta en diversos documentos internacionales: "Guías para las políticas sobre la inclusión en educación" (UNESCO, 2009); el World Report on Disability (WHO, 2011), el informe de la OCDE (2010) sobre Educación de profesores para la diversidad, etc.

En diferentes países se está haciendo una apuesta importante por la educación multicultural y el desarrollo de competencias en este campo. El Consejo Nacional de Acreditación para la función docente de EEUU, incorpora el trabajo con estudiantes de diferentes culturas, como uno de los seis estándares necesarios para la formación del profesorado (Professional Standars, 2008), porque la educación multicultural tiene efectos positivos en el desarrollo de los niños (Aslan, 2017) y que una mediación intercultural ofrece un camino seguro para la evolución y el desarrollo de la interculturalidad (Macías, 2021). La Ley Celaá, aprobada en el Congreso de los Diputados, solo cita en una ocasión la educación intercultural en su página 6 , cuando afirma que, dentro de la educación para el desarrollo sostenible y la ciudadanía mundial, hay que incluir, entre otras, la educación intercultural. 
En el ámbito de las investigaciones desarrolladas por el Equipo de Mejora Interdisciplinar de la Práctica Educativa (EMIPE) de la Facultad de Formación de Profesorado y Educación de la Universidad Autónoma de Madrid (UAM), este trabajo se propone evaluar las actitudes hacia el hecho multicultural en una muestra de estudiantes de Grado de Magisterio de Educación Infantil, Primaria y Doble grado.

\section{Metodología}

Se desarrolló una investigación con enfoque cuantitativo apoyado en un único método de recogida de datos, con carácter de escala ordinal tipo Likert, a partir de la Escala de Actitudes hacia el Hecho Multicultural de Ortega y Mínguez (1991), basado en un diseño no experimental, exploratorio, longitudinal y descriptivo.

\subsection{Participantes}

Se selecciona como población diana a estudiantes que cursan Grado de Magisterio en la Universidad Autónoma de Madrid, lo que constituye una muestra de 1170 estudiantes ( $\mathrm{n}=$ 1170). Los participantes fueron seleccionados mediante un muestreo no probabilístico entre los años 2017 y 2020. Se estimaron como criterios de inclusión de la muestra, el tener una edad igual o superior a los 18 años y estudiar alguno de los grados de Magisterio. Se considera que la mayoría de los estudiantes, por la edad y la carrera que han elegido, tienen estándares culturales muy parecidos, es decir, formas específicas de percibir, interpretar, juzgar y comportarse de una misma cultura. Por otro lado, como criterios de exclusión se estableció la dificultad idiomática y/o cognitiva y no querer participar en el estudio.

Para un mejor entendimiento de los datos, conviene mostrar inicialmente la prevalencia del género femenino frente al masculino ( $73.9 \%$ de mujeres y un $26.1 \%$ de hombres). La mayoría de los participantes son de nacionalidad española 92.9\%. Respecto al género la muestra en su mayoría es femenina ( $95.8 \%$ mujeres y $4.2 \%$ varones), la edad de la muestra se sitúa entre los 18-25 años (95.8\%). Respecto a la titulación el 80.4\% cursa el Grado de maestro en educación primaria, el grado de maestro en educación infantil es de $13.8 \%$ y tan solo un $5.8 \%$ cursan el doble grado de primaria-infantil.

\subsection{Instrumento}

La recogida de datos se realizó utilizando la Escala de Actitudes hacia el Hecho Multicultural de Ortega y Mínguez (1991). Esta escala fue construida para la valoración de las actitudes hacia el hecho multicultural en el contexto español, eliminado el condicionamiento del entorno sociocultural que rodea la construcción de estos instrumentos. Consta de 13 ítems configurados partiendo de las tres dimensiones en la actitud: pluralidad cultural, uniformidad y promoción-defensa. Su coeficiente de correlación biserial (rb) se sitúa por encima del 0.40 y su consistencia interna es de 0.77 . Ante cada uno de los ítems se ha de manifestar el grado de acuerdo en una escala Likert de 5 puntos, graduada desde Muy de acuerdo (1) a Muy en desacuerdo (5).

Está configurado en torno a tres dimensiones en la actitud hacia el hecho multicultural: Factor I (Pluralidad de culturas). El Factor II (Uniformidad) remite a una perspectiva unitaria de la organización administrativa y cultural del Estado y un Factor III (Promoción-Defensa), en el cual se engloban aquellos ítems que contemplan la defensa y promoción de las minorías étnicas y de la identidad de cada comunidad, pueblo e individuo.

La viabilidad del uso de este instrumento está avalada por la recomendación de sus autores al precisar que esta escala es viable en aquellas comunidades del Estado que no sean bilingües como es el caso de la Comunidad de Madrid. El Factor I "Pluralidad de culturas" se 
compone de los ítems 1, 2, 4, 7, 8 y 11, siendo la puntuación mínima 6 y máxima 30, el factor II "Uniformidad", de los ítems 3, 6 y 9, entre 3 y 15 puntos y por último, el factor III "Promoción y defensa" aparece en los ítems 5, 10, 12 y 13, teniendo una horquilla de puntuación entre los 4 y los 20 puntos. Por tanto, de forma cuantitativa, la horquilla de resultados se establece entre los 13 puntos de puntuación mínima, con una actitud positiva y los 65 puntos, que hace referencia a una actitud muy negativa.

\subsection{Procedimiento}

El instrumento fue aplicado de manera presencial, previa información de los objetivos y características generales de la investigación destacando el carácter voluntario, anónimo y confidencial del estudio.

El cuestionario fue presentado mediante la aplicación Google-Forms a través de un enlace compartido dentro de la Plataforma Moodle generalizada para la enseñanza, en las universidades españolas. Se obtuvo el consentimiento verbal de los participantes, conforme a los principios establecidos en el Reglamento (UE) 2016/679 del Parlamento Europeo y del Consejo de 27 de abril de 2016, relativo a la protección de las personas físicas.

El cuestionario fue presentado en una sesión habitual de clases, facilitando a los participantes una hora para su cumplimentación. Adicionalmente, los participantes recibieron información anexa sobre lenguas territoriales habladas en España, dada la presencia de personas no autóctonas entre los participantes.

\subsection{Análisis estadístico}

El análisis estadístico se realiza con el paquete estadístico SPSS ${ }^{\circledR}$ versión 22 para Windows. En todos los contrastes de hipótesis se considera un nivel de significación de 0,05. En primer lugar, se estudió la normalidad de la distribución muestral mediante el test de KolmogorovSmirnov. En segundo lugar, se verificó la validez y fiabilidad del cuestionario con los métodos más utilizados por los investigadores sociales (Cea, 2001; Mcmillan, 2005): análisis de fiabilidad alfa de Cronbach y alfa de Cronbach si se elimina un elemento. En tercer lugar, se realiza estadística descriptiva, las variables se resumen (cualitativas) con frecuencias y porcentajes, así como las variables cuantitativas en medias y desviación típica. Las variables ordinales se dan tanto de forma cualitativa como cuantitativa para una mejor compresión del lector. Finalmente, para la comparación de las competencias, se utilizan tablas de contingencia, en concreto el test de Chi cuadrado, una vez validados los requisitos. En caso de no cumplirse estos requisitos se realiza la prueba exacta de Fisher.

\section{Resultados y discusión}

\subsection{Fiabilidad y consistencia del instrumento}

En la Tabla 1 se verifica que el cuestionario alcanzó un buen nivel de fiabilidad para el objetivo de investigación propuesto y para el uso que se iba a hacer de la información como indicador para promover el debate informado. Se observa un $\alpha=0,818$ alta, con los 13 elementos del cuestionario. Si se subdivide por factores los resultados siguen siendo altos: Factor I "Pluralidad de culturas" ( $\alpha=.690)$; Factor II "Uniformidad"( $\alpha=.831)$ y Factor III "Promoción y defensa" ( $\alpha=.474)$.

En cuanto al análisis factorial, el cuestionario es coherente y sus variables están bien diseñadas y agrupadas. La prueba KMO (Kaiser-Meyer-Olkin) y el test de esfericidad de Bartlett evaluaron la aplicabilidad del análisis factorial. Los estadísticos muestran una 
adecuación muestral aceptable $(\mathrm{KMO}=.845$; Bartlett $=8713,71, \mathrm{p}=.0001)$, por lo que se procedió a realizar el análisis factorial exploratorio de la escala.

Utilizando como método de extracción mínimos cuadrados generalizados, se encontraron tres componentes con autovalores iniciales mayores a uno que acumulaban el $63.69 \%$ de la varianza. El factor 1 recoge el mayor porcentaje de varianza con un $44.87 \%$. Respecto a la matriz factorial rotada, se utilizó el método ortogonal de rotación denominado Varimax con normalización de Kaiser (convergió en nueve itinerarios). La matriz de componentes rotadas y la saturación de los ítems. Todos obtuvieron pesos factoriales superiores a 0.30 (Bandalos y Finney, 2010).

\section{Tabla 1}

Análisis de validez y fiabilidad del instrumento

\begin{tabular}{cccc}
\hline Competencias lingüísticas & a de Cronbach & $\begin{array}{c}\text { a de Cronbach basada } \\
\text { en los elementos } \\
\text { tipificados }\end{array}$ & $\begin{array}{c}\text { Número de } \\
\text { elementos }\end{array}$ \\
\hline Cuestionario General & 0,818 & 0,884 & 6 \\
\hline Factor I "Pluralidad de culturas" & 0,690 & 0,734 & 3 \\
\hline Factor II "Uniformidad" & 0,831 & 0,836 & 4 \\
\hline Factor III "Promoción y & 0,474 & 0,570 & 6 \\
defensa" & & & 0 \\
\hline
\end{tabular}

Fuente: Elaboración propia

Se realiza estadística descriptiva de los ítems recogidos en el cuestionario (Tabla 2). Se sintetizan los datos y se señalan los porcentajes, así como la media de cada ítem para una mejor valoración. La ponderación de la escala Likert fue de 1 al 5 , siendo 5 "Muy de acuerdo" y 1 "Muy en desacuerdo".

Tabla 2

Estadística descriptiva a nivel de receptor

\begin{tabular}{lcccccc}
\hline \multicolumn{1}{c}{ ítem } & $\begin{array}{c}\text { Muy } \\
\text { positivo }\end{array}$ & Positivo & Indiferente & Negativo & Muy & Media \\
& & & & Negativo & \\
(SD)
\end{tabular}


3. La utilización del castellano, como única lengua oficial en todo el territorio del Estado Español, la considero:

9. La promoción de la imagen de un Estado uniforme en su lengua, en su cultura $y$ en su forma de organización político-administrativa, la considero: $\begin{array}{llllll}97.8 \% & 0.20 \% & 0.70 \% & 1.10 \% & 0.30 \% & 1.05\end{array}$

$\begin{array}{llllll}0.80 \% & 97.4 \% & 0.20 \% & 1.50 \% & 0.20 \% & 2.02\end{array}$

\begin{tabular}{lcccccc}
\hline Item & $\begin{array}{c}\text { Muy de } \\
\text { acuerdo }\end{array}$ & $\begin{array}{c}\text { De } \\
\text { acuerdo }\end{array}$ & Indiferente & Desacuerdo & Muy en & Media \\
\end{tabular}

\begin{abstract}
2. Considero que la afirmación de la
propia identidad cultural lleva consigo la infravaloración de las demás.

4.Estimo que la denominada «pluralidad de nacionalidades y culturas» que constituyen el Estado Español es algo inventado que no responde a la realidad de los hechos.
\end{abstract}

5. Considero necesaria la protección de todas las minorías del Estado Español (gitanos, negros, inmigrantes, etc.) en su lengua y en su cultura como medio de conservar la identidad cultural de cada una de ellas.

6. La expresión utilizada por algunos políticos para referirse a algunas comunidades como naciones dentro del Estado Español, me resulta:

7. Estimo que la consecución de la convivencia pacífica y el respeto entre todas las culturas del Estado Español es algo ilusorio.

8. La promoción de los aspectos diferenciadores-singulares de una comunidad del Estado Español, como es la lengua propia, contribuye a la desintegración del Estado.

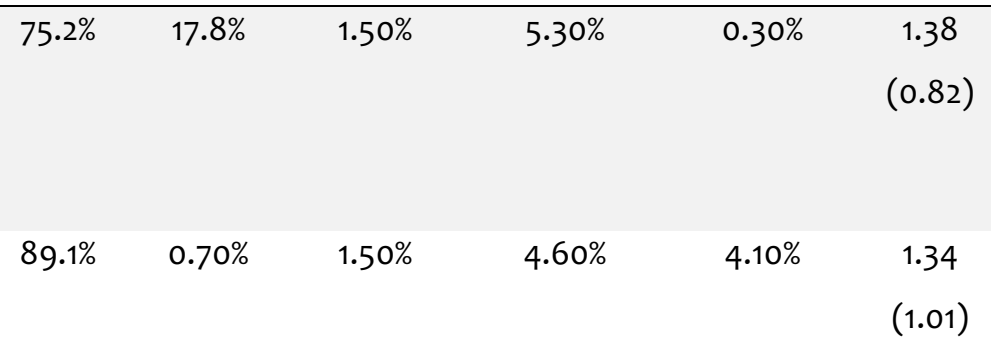

$\begin{array}{llllll}70.2 \% & 2.60 \% & 14.3 \% & 7.40 \% & 5.40 \% & 1.75\end{array}$

$\begin{array}{llllll}95.1 \% & 2.40 \% & 0.70 \% & 1.50 \% & 0.30 \% & 1.09\end{array}$

$(0.47)$
$96.8 \%$
$0.10 \%$
$0.60 \%$
$2.00 \%$
$0.50 \%$

$\begin{array}{llllll}96.3 \% & 0.20 \% & 0.30 \% & 2.80 \% & 0.30 \% & 1.10\end{array}$




\begin{tabular}{|c|c|c|c|c|c|c|}
\hline $\begin{array}{l}\text { 10. Estimo que la libre expresión de } \\
\text { la cultura de un pueblo o } \\
\text { nacionalidad es la única vía para la } \\
\text { supervivencia del mismo. }\end{array}$ & $97.4 \%$ & $0.30 \%$ & $0.10 \%$ & $2.10 \%$ & $0.30 \%$ & $\begin{array}{c}1.07 \\
(0.47)\end{array}$ \\
\hline $\begin{array}{l}\text { 11. La diversidad de culturas } \\
\text { existentes en el Estado Español, } \\
\text { lejos de ser una dificultad para la } \\
\text { convivencia entre todos, constituye } \\
\text { un patrimonio que hay que } \\
\text { conservar y promover. }\end{array}$ & $94.9 \%$ & $0.30 \%$ & $1.60 \%$ & $2.90 \%$ & $0.30 \%$ & $\begin{array}{l}1.13 \\
(0.60)\end{array}$ \\
\hline $\begin{array}{l}\text { 12. Estimo que el ser educado en la } \\
\text { propia lengua (castellano, gallego, } \\
\text { euskera, valenciano, catalán) y } \\
\text { cultura es un derecho fundamental } \\
\text { que a nadie puede ser negado. }\end{array}$ & $61.7 \%$ & $25.2 \%$ & $2.50 \%$ & $7.20 \%$ & $3.40 \%$ & $\begin{array}{c}1.65 \\
(1.05)\end{array}$ \\
\hline $\begin{array}{l}\text { 13. La promoción de las tradiciones, } \\
\text { costumbres, folklore, etc. de las } \\
\text { distintas regiones y nacionalidades } \\
\text { del Estado Español es un lujo } \\
\text { innecesario y un gasto superfluo. }\end{array}$ & $84.20 \%$ & $5.80 \%$ & $1.10 \%$ & $5.60 \%$ & $3.20 \%$ & $\begin{array}{c}1.37 \\
(0.99)\end{array}$ \\
\hline Puntuación Total & & & & & \multicolumn{2}{|c|}{$17.44(5.54)$} \\
\hline
\end{tabular}

Fuente: elaboración propia

El análisis de los datos estimados nos informa que el 94,3\% de los participantes encuestados muestra actitudes positivas hacia el factor I «Pluralidad de culturas». El 4,3\% considera como algo negativo el hecho multicultural y un $1,4 \%$ se muestra indiferente. Si bien es cierto que la mayoría de los encuestados (94,3\%) muestra una actitud positiva hacia el hecho multicultural, un análisis más pormenorizado nos revela que un 96,9\% manifiesta que es ilusorio conseguir una convivencia pacífica basada en el respeto a todas las culturas existentes en el Estado, mientras que el $2,5 \%$ se muestra en desacuerdo con dicha afirmación. Éste elevado porcentaje parece desalentador ya que los participantes desconfían de la educación, entre otros factores, como elemento facilitador de una mejor convivencia y de respeto a las diferentes culturas.

Del mismo modo, es preocupante el elevado porcentaje (96,5\%) de futuros educadores para quienes la promoción de aspectos diferenciadores-singulares de una comunidad, como es la lengua propia, contribuya a la desintegración del Estado. En la misma línea, un 95,2\% considera la diversidad de culturas existente en el Estado como un factor que aumenta la brecha en la convivencia de todos.

Respecto al segundo factor de la escala, los datos globales obtenidos muestran que los entrevistados presentan una clara aceptación a la utilización del castellano, como única lengua oficial en todo el territorio del Estado (94.8\%) o la promoción de un solo idioma. Consideran positivo la organización cultural y administrativa unificada (98.2\%). Finalmente, el 
98\% de la muestra mostró una actitud positiva hacia el realce de la imagen del país, que está unificado en su cultura y en el centro de su administración.

En cuanto al tercer factor de "promoción y defensa" de las minorías étnicas y la identidad cultural de las personas y los pueblos, el $86.9 \%$ de los encuestados expresaron su apoyo. Por el contrario, el $8.6 \%$ de los participantes piensa que es negativo y el $4,5 \%$ se muestra indiferente. Sin embargo, si realizamos un análisis más detallado de los ítems que constituyen este factor, el $90 \%$ de las personas piensa que promover tradiciones, costumbres y folclore es un lujo innecesario y un gasto extra. El 10.4\% de las personas no cree que recibir educación en su propio idioma sea un derecho básico. Del mismo modo, el 12,8\% de las personas cree que proteger el idioma y la cultura de las minorías étnicas no es un medio necesario para mantener su identidad cultural (Tabla 3 ).

Tabla 3

Estadística descriptiva. Puntuación total para cada uno de los factores de la escala

$\begin{array}{ll}\text { Puntuación Factor I } & 7.40(2.71)\end{array}$

IC95: 7.24-7.55

Puntuación Factor II

4.18(1.03)

IC95: $4.12-4.24$

Puntuación Factor III

$5.86(2.46)$

IC95: 5.72-6.00

Fuente: Elaboración propia.

Los resultados presentados en la Tabla 4 revelan una relación directa entre las preguntas del cuestionario. Esto avala una respuesta homogénea entre los participantes del estudio, ya que los ítems muestran dependencia entre sus ítems.

Por otro lado, se realiza un análisis en función de las variables sociodemográficas, observándose relación entre algunos ítems. No obstante, se debe tener en cuenta la diferencia tan notable respecto al género, predominando el sexo femenino, el grado (primaria) y la edad de los participantes (entre 18-25). Por tanto, esta interpretación está sujeta a importantes sesgos, siendo necesario buscar una mayor paridad para poder sacar conclusiones fehacientes respecto al género, grado y edad.

En el estudio realizado por Aslan (2017), con más de dos mil estudiantes turcos, futuros maestros, no encontró ninguna diferencia significativa en las variables de género, lugar de trabajo, edad, etc.., pero sí, en relación con el área de residencia, siendo los maestros de las áreas urbanas los que tienen percepciones más positivas sobre la multiculturalidad, que los que viven en zonas rurales. También encontró actitudes negativas ante el multiculturalismo. 
En el estudio de Han (2019) aparece que algunos futuros maestros piensan que el multiculturalismo divide al país y que no tienen formación en las Universidades sobre la educación multicultural. Para que se dé una auténtica educación multicultural es necesario pasar por las siguientes fases: tolerancia, aceptación, respeto y afirmación de la cultura, solidaridad y actitud crítica. En su estudio, los futuros maestros se situaban en la fase de la tolerancia y aceptación, pero no pasaban a las siguientes. Cuando se llega a una actitud crítica frente a la propia cultura, se está dando un paso muy importante con respecto a la educación multicultural.

\section{Tabla 4}

Relación entre los ítems del cuestionario y la variable sociodemográfica.

\begin{tabular}{|c|c|c|c|c|c|c|c|c|c|c|c|c|c|}
\hline Items & 1 & 2 & 3 & 4 & 5 & 6 & 7 & 8 & 9 & 10 & 11 & 12 & 13 \\
\hline 1 & & .001 & .001 & .001 & .001 & .001 & .001 & .001 & .001 & .001 & .001 & .001 & 001 \\
\hline 2 & & & .001 & .001 & .001 & .001 & .001 & .001 & .001 & .001 & .001 & .001 & .001 \\
\hline 3 & & & & .001 & .001 & .001 & .001 & .001 & .001 & .001 & .001 & .001 & .001 \\
\hline 4 & & & & & .001 & .001 & .001 & .001 & .001 & .001 & .001 & .001 & .001 \\
\hline 5 & & & & & & .001 & .001 & .001 & .001 & .001 & .001 & .001 & .001 \\
\hline 6 & & & & & & & .001 & .001 & .001 & .001 & .001 & .001 & .001 \\
\hline 7 & & & & & & & & 0.001 & .001 & .001 & .001 & .001 & .001 \\
\hline 8 & & & & & & & & & .001 & .001 & .001 & .001 & .001 \\
\hline 9 & & & & & & & & & & .001 & .001 & .001 & .001 \\
\hline 10 & & & & & & & & & & & .001 & .001 & .001 \\
\hline 11 & & & & & & & & & & & & .001 & .001 \\
\hline 12 & & & & & & & & & & & & & .001 \\
\hline Género & .001 & .562 & .884 & .001 & .001 & .001 & .450 & .001 & .004 & .044 & .001 & .001 & .001 \\
\hline Edad & .001 & .937 & .997 & .001 & .001 & .001 & .887 & .001 & .046 & .257 & .001 & .001 & .001 \\
\hline Grado & .001 & .001 & .222 & .001 & .001 & .001 & .005 & .001 & .001 & .354 & .001 & .001 & .001 \\
\hline
\end{tabular}

Fuente: Elaboración propia.

Banks (2004) considera que la educación multicultural tiene cinco dimensiones: contenidos de integración, procesos de construcción del conocimiento, disminución de sesgos hacia las diferentes culturas, pedagogía igualitaria y empoderamiento. Aunque tener formación multicultural no lleva asociado la realización y programación de actividades que trabajen la multiculturalidad en el aula. 
Parece una realidad constatable que se está lejos de transformar el concepto de multiculturalidad por el de interculturalidad y trabajar, desde los centros educativos, para que toda la sociedad adquiera una auténtica competencia intercultural. La educación multicultural contribuye a la capacidad de pensamiento crítico, a la conciencia del futuro maestro y a despertar a otras culturas entre los estudiantes universitarios (Yilmaz, 2016).

Yilmaz (2016) realizó una investigación con futuros docentes sobre el término multiculturalismo y educación multicultural y encontró que describen la educación multicultural como la base de la tolerancia social, deduciendo que las actitudes positivas de los profesores hacia la educación multicultural tienen un papel fundamental para poder vivir juntos y ser personas pacíficas y respetuosas con los demás. Sin embargo, también hacen alusión al miedo de que las diferentes culturas puedan fragmentar la unidad del país, tal y como se ha visto en la investigación que se presenta.

De esta misma investigación se dedujo que la educación multicultural consigue los siguientes objetivos: Detener la marginación, prevenir los conflictos sociales basados en las diferencias, crear una conciencia cultural, aportar respeto y armonía, crear una estructura social imparcial, incrementar la calidad de la formación docente y asegurar el logro académico.

Akar y Ulu (2016) elaboraron una escala para medir el multiculturalismo. El propósito de este estudio fue doble: desarrollar una herramienta de medición válida y confiable para medir las actitudes y opiniones de los profesores de aula con respecto al multiculturalismo y estudiar las actitudes y opiniones de los profesores de aula hacia la multiculturalidad en términos de variables de género, socioeconómicas y de devoción. Como resultado del estudio, se estableció que los candidatos a maestros de aula tenían una actitud positiva hacia la multiculturalidad, tenían suficiente conocimiento y conciencia sobre el multiculturalismo, sin embargo, el reflejo conductual de dicho conocimiento y conciencia no fue satisfactorio

Moore, Brown, Titu, Rozowa, Sivaraj y Heydari (2018) realizaron un estudio sobre la visión de la multiculturalidad, a partir de los comentarios realizados por profesores en activo y profesores en formación del que se desprendía que eran conscientes de la presión de la cultura dominante, sobre el resto de las culturas, pero no veían su implicación personal en la resolución de esta situación, desde el ámbito de la acción social.

Abd (2016) realizó un estudio para investigar el potencial de educar a los futuros profesores para el multiculturalismo crítico a través de la pedagogía dialógica. Esta experiencia demostró que esta pedagogía ayudó a los estudiantes a tener una cosmovisión sobre ciertos temas del currículo multicultural, a los que inicialmente no estaban abiertos al diálogo. Al poder contrastar sus opiniones, con opiniones contrarias, les ayudó a conocer más la realidad que les rodeaba y admitir la visión opuesta, al encuadrarse en un diálogo igualitario, donde todos los argumentos pueden ser aceptados y porque el objetivo fundamental no es llegar a un consenso, sino aprender del mismo diálogo que se produce. Este autor sostiene que la educación multicultural tiene que incluir la ética y la práxis. Todo maestro debe estar convencido de que la equidad educativa es el derecho de todos los estudiantes a ser instruidos y, por tanto, todas sus prácticas educativas se deben desarrollar en esta línea (Banks, 2012).

\section{Conclusiones}

A partir de los resultados obtenidos con la aplicación del Cuestionario-escala de actitudes hacia el hecho multicultural de Ortega y Mínguez (1991), queda de manifiesto que todavía hay mucho que hacer en cuanto a la educación multicultural y que los futuros profesores son escépticos en la creencia de que la sociedad puede tener una convivencia pacífica entre las 
diferentes culturas, lo que es más desalentador en los que tienen una responsabilidad muy importante en propiciar este clima de convivencia en las aulas.

Por otra parte, no es atractiva la idea de que no estén de acuerdo con preservar las diferentes culturas, considerando que es fundamental la unidad cultural de España y que hay que hacer todo lo posible para que esa unidad se mantenga, considerando un lujo el mantenimiento de las costumbres y tradiciones de otras culturas. Sin embargo, un porcentaje muy alto considera que la diversidad de culturas existentes en el Estado Español no supone una dificultad para la convivencia, sino que son un patrimonio que hay que conservar y promover.

La interculturalidad tiene que ser la apuesta a las sociedades multiculturales porque ésta "se fundamenta en la consideración de la diversidad humana como oportunidad de intercambio y enriquecimiento frente a la incoherencia pedagógica de la educación monocultural" (Alarcón y Márquez, 2019, p.25). Es un reto para los formadores de formadores, favorecer el desarrollo de las competencias de comportamiento intercultural, a través de la formación de valores, dentro y fuera del aula, ya que el arraigo con la comunidad de origen será un elemento fundamental para valorar lo diferente, porque no se puede querer, lo que no se conoce. Educar hacia el multiculturalismo conduce a una atmósfera de apertura e igualitarismo, a mejores relaciones interpersonales y habilidades sociales en la clase (MagenNagar y Shonfeld, 2018)

En la línea de Torres y Tarozzi (2020), afirmamos que el multiculturalismo es un nuevo desafío para las Instituciones de Educación Superior y supone una premisa en la construcción de modelos de democracias cosmopolitas y en lo que se conoce como "educación para la ciudadanía global". Se apuesta por la idea de justicia social, cuando no solo se aplica a las desigualdades de bienes materiales sino a las que se derivan de no reconocer las diferencias y la identidad cultural de los demás (Torres, 2019). Crear entornos educativos democráticos que reflejen la diversidad cultural, respeten las diferencias y las acepten como un tesoro son partes inseparables del ser humano (Aslan, 2017).

\section{Referencias}

Abd Elkader, N. (2016). Dialogic Pedagogy and Educating Preservice Teachers for Critical Multiculturalism. SAGE Open, 6, (1), 1-13. DOI: http://dx.doi.org/10.1177/2158244016628592

Akar, C., y Ulu, M. (2016). Attitudes and Opinions of Classroom Teacher Candidates Regarding Multiculturalism. Educación, 137 (2), 220-232.

Alarcón Leiva, J., y Márquez Sánchez, J. (2019). Competencias docentes interculturales. Multiculturalidad y consecuencias para la inmigración, Estudios pedagógicos, XLV (2), 7-27.

Arroyo, M. J. (2013). La educación intercultural: un camino hacia la inclusión educativa. Inclusive Education Journal, 6 (2), 144-159.

Aslan, G. (2017). Teachers and multiculturalism in Turkey. An evaluation of the competency perceptions of teachers regarding multiculturalism and their reflection of these perceptions to the classroom. Journal for Critical Education Polizy Studies, 15 (3), 361396.

Assiego, V. (jueves 15 de diciembre de 2016). Ante la inmigración, los españoles no piensan como Europa. Matroscopia, en: https://metroscopia.org/ante-la-inmigracion-losespanoles-no-piensan-como-europa/ (consulta: 21 de mayo de 2021) 
Bagan, S., , Jiménez Nuria, M. D. , y Ruiz R. (2005-2006). Actitudes del profesorado hacia la diversidad cultural. Jornades de Foment de la Investigació "Universitat Jaume I", Fòrum de recerca $n^{\circ} 11$,

http://repositori.uji.es/xmlui/bitstream/handle/10234/78807/forum_2005_12.pdf?seq uence $=1$ (consulta: 21 de mayo de 2021)

Banks, J. (1989). Approaches to Multicultural Curriculum Reform. Trotter Review, 3 (3).

Barabas, A. (2014). Multiculturalismo, pluralismo cultural y interculturalidad en el contexto de América Latina: la presencia de los pueblos originarios. Configurações, 14 (1), 11-24. DOI: https://doi.org/10.4000/configuracoes.2219

Bayona, E. (2019). España se mezcla: uno de cada seis ciudadanos ya es hijo de padres extranjeros. Púublico https://www.publico.es/sociedad/espana-mezcla-seisciudadanos-hijo-padres-extranjeros.html.

Bazán, D. (2011). Multiculturalismo y diversidad: de la opresión a la esperanza. Paulo Freire. Revista de Pedagogía Crítica, 10 (9), 35-45. DOI: https://doi.org/10.25074/07195532.9.437.

Bernabé, M. P. (2012). Pluriculturalidad, multiculturalidad e interculturalidad, conocimientos necesarios para la labor docente. Hekademos, 11, 67-76.

Boletín Oficial de las Cortes generales Senado (2020). Proyecto de Ley Orgánica por la que se modifica la Ley Orgánica 2/2006, de 3 de mayo de Educación. Núm. 113, en: http://www.senado.es (consulta: 21 de mayo de 2021)

Casares, S. (2019). Racismo en la escuela: asignatura pendiente. El Salto. https://www.elsaltodiario.com/educacion/racismo-escuela-asignatura-pendiente

Casas Castañé, M. (1999). Cambio de actitudes en contextos interculturales en Barcelona: actividades lúdicas y modificación de prejuicios. Scripta Nova. Revista Electrónica de Geografía y Ciencias Sociales, 44, 1-11.

Comisión Española de Ayuda al Refugiado (2016). Informe sobre discriminación de personas migrantes y refugiadas en España. http://www.mitramiss.gob.es/oberaxe/ficheros/documentos/Informe2018DelitosOdi o.pdf.

Consejo Económico y Social (2019). La inmigración en España: efectos y oportunidades. Informe 02|2019. Sesión ordinaria del Pleno de 20 de marzo de 2019, http://www.ces.es/documents/10180/5209150/Inf0219.pdf (consulta: 20 de mayo de 2021)

Datosmacro.com (2019), Inmigración en España. https://datosmacro.expansion.com/demografia/migracion/emigracion/espana.

Escalante, E., Repetto, A. M., y Martinello, G. (2012). Exploración y análisis de la actitud hacia la estadística en alumnos de psicología. Liberabit, 18 (1), 15-26.

Estrach, N. (2001). Las máscaras del multiculturalismo. Scripta Nova, Revista electrónica de geografía y ciencias sociales, 94 (104). http://www.ub.edu/geocrit/sn-94-104.htm

Figueredo Canosa, V., y Ortiz Jiménez, L. (2017). Formación inicial del profesorado para la inclusión de la diversidad cultural. Revista Digital de Investigación en Docencia Universitaria, 11(1), 38-61. DOI: http://dx.doi.org/10.19083/ridu.11.529.

González, O., Berríos LL., y Buxarrais, M. (2013). La sensibilidad del profesorado hacia el modelo de educación intercultural: Necesidades, situación actual y propuesta de un 
instrumento de medida. Estudios pedagógicos, 39 (2), 147-164. DOI: https://dx.doi.org/10.4067/S0718-07052013000200010

Hernández, M. (2007). Sobre los sentidos de 'multiculturalismo' e 'interculturalismo. Revista de Sociedad, Cultura y Desarrollo Sustentable Ra Ximhai, 3 (2), 429-442.

Herrera, J., Parrilla, A., Blanco, A., y Guevara, G. (2018). La Formación de Docentes para la Educación Inclusiva. Un Reto desde la Universidad Nacional de Educación en Ecuador. Revista latinoamericana de educación inclusiva, 12 (1), 21- 38.

INE (2021), España en cifras. en https://www.ine.esntxausti, N., Etxeberria, F., y Joaristi, L. (2014). ¿Coinciden las expectativas escolares de la familia y del profesorado acerca del alumnado de origen inmigrante?. Relieve, 20 (1), 1-21. DOI: http://doi.org/10.7203/relieve.20.1.3804.

Iñiguez-Berrozpe, T., Valero, D., Flecha, A., y Redondo-Sama, G. (2021). Hacia una evaluación de la eficacia intercultural en el profesorado en formación. Revista de Sociología de la Educación-RASE, 14 (2), 139-156. http://dx.doi.org/10.7203/RASE.14.2.16634

Macías Manteca, M.J. (2021). La acción transformadora de la sociedad multicultural en una nueva sociedad intercultural, mediante la implementación de la mediación y/o de sus principios y herramientas. MSC Métodos de solución de conflictos, 01(01), 133-156. https://revistamsc.uanl.mx/index.php/m/article/view/13

Magen-Nagar, N., y Shonfeld, M. (2018). Attitudes, Openness to Multiculturalism, and Integration of Online Collaborative Learning. Educational Technology \& Society. Journal of International Forum of Educational Technology \& Society and IEEE Learning Technology Task Force, 21 (3), 1.

Ministerio del Interior (2020. Informe sobre la evolución de los delitos de odio en España. Secretaría de Estado de Seguridad.

Moldes-Anaya, S., Jiménez Aguilar, F., y Jiménez Bautista, F. (2018). Actitudes hacia la inmigración en España a través de la Encuesta Social Europea. OBETS. Revista de Ciencias Sociales, 13 (1), 93-119.

Montes, B. (2008). Discriminación, prejuicio, estereotipos: conceptos fundamentales, historia de su estudio y el sexismo como nueva forma de prejuicio. Iniciación a la Investigación Revista Electrónica de la Universidad de Jaén, 3, 1-16.

Moore Mensah, F., Brown, J. T., y Rozowa, P. (2018). Preservice and Inservice Teachers' Ideas of Multiculturalism: Explorations Across Two Science Methods Courses in Two Different Contexts. Journal of Science Teacher Education, 29 (2), 128147. DOI: $10.1080 / 1046560 X .2018 .1425820$

OCDE (2010). Educating teachers for diversity: Meeting the challenge. OCDE.

Olivé, L. (2004). Interculturalismo y justicia social. UNAM.

Ortega, P., y Mínguez R. (1991). Actitudes hacia el hecho multicultural. Revista Interuniversitaria de Teoría de la Educación, 3 (10), 47-57. DOI: http://dx.doi.org/10.14201/teri.2913.

Padua, J., y Ahman, I. (1979). Escalas para la medición de actitudes, en Jorge Padua (ed), Técnicas de investigación aplicadas a las ciencias sociales, (pp. 160-231). FALTA LA EDITORIAL.

Professional Standards for the accreditation of teacher preparation institutions (2008). Experiences working with diverse students http://www.ncate.org._Reglamento (UE) 
2016/679 del Parlamento Europeo y del Consejo de 27 de abril de 2016 relativo a la protección de las personas físicas en lo que respecta al tratamiento de datos personales y a la libre circulación de estos datos y por el que se deroga la Directiva 95/46/CE (Reglamento general de protección de datos). https://www.boe.es/doue/2016/119/L00001-00088.pdf (consulta: 21 de mayo de 2021)

Rinken, S. (2015). Actitudes hacia la inmigración y los inmigrantes: ¿En qué es España excepcional?. Migraciones, 37, 53-74. DOI: https://doi.org/10.14422/mig.i37.y2015.003

Rodríguez, A., y Fernández, A. (2018). Agentes educativos y multiculturalidad en el aula. Educação e Pesquisa, 44, 1-21. DOI: http://dx.doi.org/10.1590/S16784634201844188506

Torres Rivera, E. (2019). Deconstruyendo la universalidad de la justicia social: una perspectiva multicultural hacia nuevos horizontes. Revista Interamericana de Psicología, 53(1), pp. $1-7$.

Torre, C., y Tarozzi, M. (2020). Multiculturalism in the world system: towards a social justice model of inter/multicultural education. Globalisation, Societies and Education, 18 (1), 7-18. DOI: http://doi.org/10.1080/14767724.2019.1690729

UNESCO (2009). Policy guidelines on inclusion in education. UNESCO.

World Health Organization (2011). World report on disability. WHO.

Yilmaz, F. (2016). Multiculturalism and Multicultural Education: A Case Study of Teacher Candidates' Perceptions. Cogent Education, 3 (1), 1-13. DOI: http://dx.doi.org/10.1080/2331186X.2016.1172394 